PROCEEDINGS OF THE

AMERICAN MATHEMATICAL SOCIETY

Volume 133, Number 8, Pages 2387-2393

S 0002-9939(05)08132-3

Article electronically published on March 21, 2005

\title{
ON MORDELL-TORNHEIM ZETA VALUES
}

\author{
HIROFUMI TSUMURA
}

(Communicated by Wen-Ching Winnie Li)

\begin{abstract}
We prove that the Mordell-Tornheim zeta value of depth $r$ can be expressed as a rational linear combination of products of the Mordell-Tornheim zeta values of lower depth than $r$ when $r$ and its weight are of different parity.
\end{abstract}

\section{INTRODUCTION}

For complex numbers $s_{1}, s_{2}, \ldots, s_{r}, s$, Matsumoto defined the Mordell-Tornheim $r$-fold zeta function $\zeta_{M T, r}\left(s_{1}, s_{2}, \ldots, s_{r} ; s\right)$ by

$$
\sum_{m_{1}, m_{2}, \ldots, m_{r}=1}^{\infty} \frac{1}{m_{1}^{s_{1}} m_{2}^{s_{2}} \cdots m_{r}^{s_{r}}\left(m_{1}+\cdots+m_{r}\right)^{s}},
$$

where the sum is over $r$-tuples of positive integers (see [4, 5]). He showed that this function can be continued meromorphically to the whole $r$-dimensional complex space. The origin of this function goes back to Tornheim and Mordell. Tornheim investigated the properties of $\zeta_{M T, 2}\left(k_{1}, k_{2} ; k\right)$ for positive integers $k_{1}, k_{2}, k$ and discovered some relations (see [8]). Later Mordell independently considered $\zeta_{M T, 2}(k, k ; k)$ for any even positive integer $k$ and also studied the values

$$
\sum_{m_{1}, m_{2}, \ldots, m_{r}=1}^{\infty} \frac{1}{m_{1} m_{2} \cdots m_{r}\left(m_{1}+\cdots+m_{r}+a\right)}
$$

with $a>r$ (see [6], see also [7]). By using this result, Hoffman gave some evaluation formulas for $\zeta_{M T, r}(1, \ldots, 1 ; k)$ for any positive integer $k$ (see [1]).

In the present paper, we aim to consider $\zeta_{M T, r}\left(k_{1}, \ldots, k_{r} ; k\right)$ for positive integers $k_{1}, \ldots, k_{r}, k$ with $k \geq 2$. We call it the Mordell-Tornheim zeta value of depth $r$ and of weight $\sum_{j=1}^{r} k_{j}+k$ as well as the multiple zeta values defined by

$$
\sum_{1 \leq m_{1}<m_{2}<\cdots<m_{r}} \frac{1}{m_{1}^{k_{1}} m_{2}^{k_{2}} \cdots m_{r}^{k_{r}}}
$$

for positive integers $k_{1}, k_{2}, \ldots, k_{r}$ with $k_{r} \geq 2$ (see, for example, [1]).

Received by the editors July 7, 2003 and, in revised form, March 20, 2004

2000 Mathematics Subject Classification. Primary 40B05; Secondary 11M06, 30B99, 33E20, $40 \mathrm{~A} 05$.

Key words and phrases. Tornheim's double series, multiple zeta values, Riemann's zeta function, uniformly convergent series. 
The main aim of this paper is to prove

Theorem 1.1. The Mordell-Tornheim zeta value of depth $r$ with $r \geq 2$ and of weight $w$ can be expressed as a rational linear combination of products of the Mordell-Tornheim zeta values of lower depth than $r$, when its depth $r$ and its weight $w$ are of different parity.

Note that this theorem is an analogue of the result on multiple zeta values, which was proved by Zagier (see [3]), and has recently been proved in a different method by the author (see [9]).

The case $r=2$ of Theorem 1.1 was proved in [8] and the explicit formulas were given in [2]. The case $r=3$ was proved in [10].

The author greatly thanks the referee for his (or her) helpful comments.

\section{Preliminaries}

We use the same notation as in 9 . Let $\mathbb{N}$ be the set of natural numbers, $\mathbb{N}_{0}=\mathbb{N} \cup\{0\}, \mathbb{Z}$ the ring of rational integers, $\mathbb{Q}$ the field of rational numbers, and $\mathbb{R}$ the field of real numbers. Throughout this paper we fix $\delta \in \mathbb{R}$ with $\delta>0$. For $u \in \mathbb{R}$ with $1 \leq u \leq 1+\delta$, we define

$$
\phi(s ; u)=\sum_{m=1}^{\infty} \frac{(-u)^{-m}}{m^{s}} \quad(s \in \mathbb{R}) .
$$

If $u>1$, then $\phi(s ; u)$ is convergent for any $s \in \mathbb{R}$. In the case when $u=1$, let $\phi(s)=\phi(s ; 1)=\left(2^{1-s}-1\right) \zeta(s)$, where $\zeta(s)$ is the Riemann zeta function. Corresponding to $\phi(s ; u)$, we define a set of numbers $\left\{\varepsilon_{m}(u)\right\}$ by

$$
F(x ; u)=\frac{(1+u) e^{x}}{e^{x}+u}=\sum_{m=0}^{\infty} \varepsilon_{m}(u) \frac{x^{m}}{m !} \quad(|x|<\pi) .
$$

Note that

$$
\varepsilon_{2 j}(1)=0 \quad(j \in \mathbb{N}) .
$$

It follows from $(2.2)$ that if $u \in[1,1+\delta]$, then

$$
\liminf _{m \rightarrow \infty}\left(\frac{\left|\varepsilon_{m}(u)\right|}{m !}\right)^{-1 / m} \geq \pi \quad \text { and } \quad \frac{\left|\varepsilon_{n}(u)\right|}{n !} \leq \frac{M}{\gamma^{n}} \quad\left(n \in \mathbb{N}_{0}\right)
$$

for any $\gamma$ with $0<\gamma<\pi$, where $M$ is the constant independent of $n$ and $u$.

$$
\phi(-k ; u)=-\frac{1}{1+u} \varepsilon_{k}(u)
$$

for $k \in \mathbb{N}_{0}$ and $u \in(1,1+\delta]$ (see [9], Lemma 1). For simplicity we let

$$
\mathcal{R}_{n}\left(\theta ; k_{1}, \ldots, k_{n} ; u\right)=\sum_{m_{1}, \ldots, m_{n}=1}^{\infty} \frac{(-u)^{-\sum_{\nu=1}^{n} m_{\nu}} e^{i\left(\sum_{\nu=1}^{n} m_{\nu}\right) \theta}}{m_{1}^{k_{1}} \cdots m_{n}^{k_{n}}}
$$

for $k_{1}, \ldots, k_{n} \in \mathbb{N}, u \in[1,1+\delta]$ and $\theta \in \mathbb{R}$, where $i=\sqrt{-1}$. We define $\lambda_{j}=$ $\left(1+(-1)^{j}\right) / 2$ for $j \in \mathbb{Z}$ and

$$
H(\theta ; k ; u)=\mathcal{R}_{1}(\theta ; k ; u)-\sum_{\nu=0}^{k} \phi(k-\nu ; u) \lambda_{k-\nu} \frac{(i \theta)^{\nu}}{\nu !}
$$


for $k \in \mathbb{N}, \theta \in \mathbb{R}$ and $u \in[1,1+\delta]$. It follows from (2.5) and (2.6) that

$$
H(\theta ; k ; u)=\sum_{\nu=0}^{k} \phi(k-\nu ; u) \lambda_{k-\nu+1} \frac{(i \theta)^{\nu}}{\nu !}-\frac{1}{1+u} \sum_{N=1}^{\infty} \varepsilon_{N}(u) \frac{(i \theta)^{N+k}}{(N+k) !}
$$

when $u \in(1,1+\delta]$. From (2.4) we can see that each side of (2.8) is uniformly convergent with respect to $u \in(1,1+\delta]$ if $\theta \in(-\pi, \pi)$ and $k \in \mathbb{N}$. So (2.8) holds for $u=1$. Hence it follows from (2.3) that if $k$ is even (resp. odd), then $H(\theta ; k ; 1)$ is odd (resp. even) function. Namely

$$
H(-\theta ; k ; 1)=(-1)^{k+1} H(\theta ; k ; 1) .
$$

For $k_{1}, \ldots, k_{r} \in \mathbb{N}, \theta \in \mathbb{R}$ and $u \in[1,1+\delta]$, we define

$$
G\left(\theta ; k_{1}, \ldots, k_{r} ; u\right)=\prod_{j=1}^{r} H\left(\theta ; k_{j} ; u\right)
$$

By (2.9), we have

$$
G\left(-\theta ; k_{1}, \ldots, k_{r} ; 1\right)=(-1)^{\sum_{j=1}^{r} k_{j}+r} G\left(\theta ; k_{1}, \ldots, k_{r} ; 1\right) .
$$

Now we prepare some notation. Let

$$
\Delta_{s}=\left\{J=\left\{j_{1}, \ldots, j_{s}\right\} \mid J \subset\{1,2, \ldots, r\}\right\} \text { and } \Delta=\bigcup_{s=1}^{r-1} \Delta_{s} .
$$

For $J=\left\{j_{1}, \ldots, j_{s}\right\} \in \Delta$, let $\bar{J}=\{1, \ldots, r\} \backslash J$. Let $\Omega(u)$ be the $\mathbb{Q}$-algebra generated by $\left\{\phi(2 j ; u) \mid j \in \mathbb{N}_{0}\right\}$. Note that $\Omega(1)=\mathbb{Q}\left[\pi^{2}\right]$ because $\phi(s ; 1)=$ $\left(2^{1-s}-1\right) \zeta(s)$.

By (2.7) and (2.10), we have the expansion

$$
\begin{aligned}
G\left(\theta ; k_{1}, \ldots, k_{r} ; u\right) \\
=\mathcal{R}_{r}\left(\theta ; k_{1}, \ldots, k_{r} ; u\right) \\
\quad+\sum_{J=\left\{j_{1}, \ldots, j_{s}\right\}} \sum_{\mu}^{*} B(J ; \mu ; u) \frac{(i \theta)^{\mu}}{\mu !} \mathcal{R}_{s}\left(\theta ; k_{j_{1}}, \ldots, k_{j_{s}} ; u\right) \\
\quad+\sum_{\nu}^{*} C(\nu ; u) \frac{(i \theta)^{\nu}}{\nu !},
\end{aligned}
$$

where the sums $\sum_{\mu}{ }^{*}$ and $\sum_{\nu}{ }^{*}$ are taken over all $\mu, \nu \in \mathbb{N}_{0}$ with

$$
0 \leq \mu \leq \sum_{j \in \bar{J}} k_{j}, 0 \leq \nu \leq \sum_{l=1}^{r} k_{l}
$$

respectively, and $B(J ; \mu ; u), C(\nu ; u) \in \Omega(u)$. Note that

$$
B(J ; \mu ; 1), C(\nu ; 1) \in \Omega(1)=\mathbb{Q}\left[\pi^{2}\right] .
$$




\section{Some Lemmas}

We define the multiple series

$$
\rho_{n}\left(k_{1}, \ldots, k_{n} ; s ; u\right)=\sum_{m_{1}, m_{2}, \ldots, m_{n}=1}^{\infty} \frac{(-u)^{-\sum_{\nu=1}^{n} m_{\nu}}}{m_{1}^{k_{1}} \cdots m_{n}^{k_{n}}\left(m_{1}+\cdots+m_{n}\right)^{s}}
$$

for $k_{1}, \ldots, k_{n} \in \mathbb{N}, s \in \mathbb{R}$ and $u \in[1,1+\delta]$. In particular when $n=1$, we have $\rho_{1}\left(k_{1} ; s ; u\right)=\phi\left(k_{1}+s ; u\right)$. If $u \in(1,1+\delta]$, then

$$
\mathcal{R}_{n}\left(\theta ; k_{1}, \ldots, k_{n} ; u\right)=\sum_{N=0}^{\infty} \rho_{n}\left(k_{1}, \ldots, k_{n} ;-N ; u\right) \frac{(i \theta)^{N}}{N !} .
$$

For $N \in \mathbb{Z}$ and $u \in(1,1+\delta]$ we define

$$
\begin{aligned}
& \mathcal{A}_{N}\left(k_{1}, \ldots, k_{r} ; u\right) \\
& \quad=\rho_{r}\left(k_{1}, \ldots, k_{r} ;-N ; u\right) \\
& \quad+\sum_{\substack{J=\left\{j_{1}, \ldots, j_{s}\right\} \\
\in \in \Delta}} \sum_{\mu}^{*} B(J ; \mu ; u)\left(\begin{array}{c}
N \\
\mu
\end{array}\right) \rho_{s}\left(k_{j_{1}}, \ldots, k_{j_{s}} ; \mu-N ; u\right) .
\end{aligned}
$$

Furthermore we define

$$
\begin{aligned}
\widetilde{\mathcal{A}}_{N} & \left(k_{1}, \ldots, k_{r} ; u\right) \\
\quad & = \begin{cases}\mathcal{A}_{N}\left(k_{1}, \ldots, k_{r} ; u\right)+C(N ; u) & \left(\text { if } 0 \leq N \leq \sum_{j=1}^{r} k_{j}\right), \\
\mathcal{A}_{N}\left(k_{1}, \ldots, k_{r} ; u\right) & \text { (otherwise) }\end{cases}
\end{aligned}
$$

Note that if $N \leq-1$, then we can define

$$
\widetilde{\mathcal{A}}_{N}\left(k_{1}, \ldots, k_{r} ; 1\right)=\lim _{u \rightarrow 1} \widetilde{\mathcal{A}}_{N}\left(k_{1}, \ldots, k_{r} ; u\right) .
$$

By combining (2.12), (3.2) and (3.4), we have

Lemma 3.1. With the above notation,

$$
G\left(\theta ; k_{1}, \ldots, k_{r} ; u\right)=\sum_{N=0}^{\infty} \widetilde{\mathcal{A}}_{N}\left(k_{1}, \ldots, k_{r} ; u\right) \frac{(i \theta)^{N}}{N !} .
$$

By (2.2), (2.8), (2.10) and (3.5), we have

Lemma 3.2. With the above notation and for $u \in(1,1+\delta]$,

$$
\liminf _{N \rightarrow \infty}\left(\frac{\left|\widetilde{\mathcal{A}}_{N}\left(k_{1}, \ldots, k_{r} ; u\right)\right|}{N !}\right)^{-1 / N} \geq \pi \text { and } \frac{\left|\widetilde{\mathcal{A}}_{n}\left(k_{1}, \ldots, k_{r} ; u\right)\right|}{n !} \leq \frac{\widetilde{M}}{\gamma^{n}}
$$

for any $\gamma \in(0, \pi)$ and $n \in \mathbb{N}_{0}$, where $\widetilde{M}(>0)$ is independent of $n$ and $u$. Further

$$
\lim _{u \rightarrow 1} \widetilde{\mathcal{A}}_{N}\left(k_{1}, \ldots, k_{r} ; u\right) \lambda_{N+1+r+\sum_{j=1}^{r} k_{j}}=0
$$

for $N \in \mathbb{N}_{0}$.

For simplicity we let

$$
\mathcal{S}_{n}^{p}\left(\theta ; k_{1}, \ldots, k_{n} ; s ; u\right)=\sum_{m_{1}, \ldots, m_{n}=1}^{\infty} \frac{(-u)^{-\sum_{\nu=1}^{n} m_{\nu}} \sin ^{(p)}\left(\left(\sum_{\nu=1}^{n} m_{\nu}\right) \theta\right)}{m_{1} k_{1} \cdots m_{n}{ }^{k_{n}}\left(m_{1}+\cdots+m_{n}\right)^{s}}
$$

for $p \in \mathbb{N}_{0}, s \in \mathbb{R}, k_{1}, \ldots, k_{n} \in \mathbb{N}$ and $u \in[1,1+\delta]$, where we denote the $l$ th derivative of a function $f(\theta)$ by $f^{(l)}(\theta)$. Then we have the following lemma. 
Lemma 3.3. With the above notation and for $l \in \mathbb{N}$ with $l \geq 2$,

$$
\begin{aligned}
& \mathcal{S}_{r}^{p}\left(\theta ; k_{1}, \ldots, k_{r} ; l+p ; u\right) \\
& +\sum_{\substack{J=\left\{j_{1}, \ldots, j_{s}\right\} \\
\in \leq}} \sum_{\mu}^{*} B(J ; \mu ; u)(-1)^{\mu} \sum_{\sigma=0}^{\mu}\left(\begin{array}{c}
l+p+\mu-1-\sigma \\
\mu-\sigma
\end{array}\right) \\
& \quad \times \mathcal{S}_{s}^{(\sigma+p)}\left(\theta ; k_{j_{1}}, \ldots, k_{j_{s}} ; l+p+\mu-\sigma ; u\right) \frac{(-\theta)^{\sigma}}{\sigma !} \\
& +i^{p-1} \sum_{\nu}^{*} C(\nu ; u) \lambda_{\nu+l+1} \frac{(i \theta)^{\nu+l+p}}{(\nu+l+p) !} \\
& =i^{p-1} \sum_{n=-l-p}^{\infty} \widetilde{\mathcal{A}}_{n}\left(k_{1}, \ldots, k_{r} ; u\right) \lambda_{n+l+1} \frac{(i \theta)^{n+l+p}}{(n+l+p) !} .
\end{aligned}
$$

Proof. It is known that

$$
\begin{aligned}
& \sum_{\nu=0}^{b}\left(\begin{array}{c}
a-1+b-\nu \\
b-\nu
\end{array}\right) \frac{(-\theta)^{\nu}}{\nu !} \frac{\sin ^{(\nu+p)}(\theta x)}{x^{a+b-\nu}} \\
& =i^{p-1} \sum_{N \geq 0}\left(\begin{array}{c}
a-1+b-N \\
b
\end{array}\right) \frac{(i \theta)^{N}}{N !} \lambda_{p+1+N} x^{-a-b+N}
\end{aligned}
$$

for $a, b \in \mathbb{N}_{0}$ (see [9], (2.16)). We assume that $u \in(1,1+\delta]$ and apply (3.10) with $a=l+p$ and $b=0$. Then we obtain

$$
\begin{aligned}
& \mathcal{S}_{r}^{p}\left(\theta ; k_{1}, \ldots, k_{r} ; l+p ; u\right) \\
& \quad=i^{p-1} \sum_{N=0}^{\infty} \rho_{r}\left(k_{1}, \ldots, k_{r} ; l+p-N ; u\right) \lambda_{p+1+N} \frac{(i \theta)^{N}}{N !} .
\end{aligned}
$$

Applying (3.10) with $a=l+p$ and $b=\mu$, we have

$$
\begin{aligned}
& \sum_{J=\left\{j_{1}, \ldots, j_{s}\right\}} \sum_{\mu}^{*} B(J ; \mu ; u) \sum_{\sigma=0}^{\mu}\left(\begin{array}{c}
l+p+\mu-1-\sigma \\
\mu-\sigma
\end{array}\right) \\
& \times \mathcal{S}_{s}^{\sigma+p}\left(\theta ; k_{j_{1}}, \ldots, k_{j_{s}} ; l+p+\mu-\sigma ; u\right) \frac{(-\theta)^{\sigma}}{\sigma !} \\
& =i^{p-1} \sum_{N=0}^{\infty} \sum_{\substack{J=\left\{j_{1}, \ldots, j_{s}\right\} \\
\in \in}} \sum_{\mu}^{*} B(J ; \mu ; u)\left(\begin{array}{c}
l+p+\mu-1-N \\
\mu
\end{array}\right) \\
& \quad \times \rho_{s}\left(k_{j_{1}}, \ldots, k_{j_{s}} ; l+p+\mu-N ; u\right) \lambda_{p+1+N} \frac{(i \theta)^{N}}{N !} .
\end{aligned}
$$

Let $n=N-l-p$. By (3.3), (3.4), (3.11), (3.12) and using the well-known relation

$$
\left(\begin{array}{c}
-X \\
j
\end{array}\right)=(-1)^{j}\left(\begin{array}{c}
X+j-1 \\
j
\end{array}\right)
$$

we obtain (3.9). 


\section{Proof of Theorem 1.1}

In this section, we give the proof of Theorem 1.1. Let $\mathcal{M} \mathcal{T}_{n}$ be the $\mathbb{Q}$-algebra generated by the Mordell-Tornheim zeta values of depth lower than or equal to $n$. Assume $r \geq 2$. Then we aim to prove that the Mordell-Tornheim zeta values of depth $r$ and of weight $w$ belongs to $\mathcal{M T}_{r-1}$ if $r$ and $w$ are of different parity.

By (3.6), we can see that (3.9) is uniformly convergent with respect to $u \in$ $(1,1+\delta]$ if $l+p \geq 2$ and $\theta \in[-\pi, \pi]$. So we let $\theta=\pi$ and $u \rightarrow 1$ in both sides of (3.9). For simplicity we let

$$
\begin{aligned}
\mathcal{F}^{p}\left(k_{1}, \ldots, k_{r} ; l\right)= & \sum_{\substack{J=\left\{j_{1}, \ldots, j_{s}\right\} \\
\in \Delta}} \sum_{\mu}^{*}(-1)^{\mu} B(J ; \mu ; 1) \sum_{\sigma=0}^{\mu}\left(\begin{array}{c}
l+p+\mu-1-\sigma \\
\mu-\sigma
\end{array}\right) \\
& \times \mathcal{S}_{s}^{\sigma+p}\left(\pi ; k_{j_{1}}, \ldots, k_{j_{s}} ; l+p+\mu-\sigma ; 1\right) \frac{(-\pi)^{\sigma}}{\sigma !} \\
& +i^{p-1} \sum_{\nu}^{*} C(\nu ; 1) \lambda_{\nu+l+1} \frac{(i \pi)^{\nu+l+p}}{(\nu+l+p) !} .
\end{aligned}
$$

By the facts $\mathcal{S}_{s}^{2 m}\left(\pi ; k_{j_{1}}, \ldots, k_{j_{s}} ; k ; 1\right)=0, \mathcal{S}_{s}^{2 m+1}\left(\pi ; k_{j_{1}}, \ldots, k_{j_{s}} ; k ; 1\right) \in \mathcal{M T}_{s}$ and (2.13), we have

$$
\pi^{p-1} \mathcal{F}^{p}\left(k_{1}, \ldots, k_{r} ; l\right) \in \mathcal{M T}_{r-1} \quad(p=0,1),
$$

when $l+p \geq 2$, because $\pi^{2} \subset \mathcal{M} \mathcal{T}_{1} \subset \mathcal{M} \mathcal{T}_{r-1}$ for $r \geq 2$. Suppose $l \equiv \sum_{\eta=1}^{r} k_{\eta}+$ $r(\bmod 2)$ and $l+p \geq 2$. It follows from (3.7) that

$$
\begin{aligned}
& \mathcal{S}_{r}^{p}\left(\pi ; k_{1}, \ldots, k_{r} ; l+p ; 1\right)+\mathcal{F}^{p}\left(k_{1}, \ldots, k_{r} ; l\right) \\
& \quad=i^{p-1} \sum_{\mu=0}^{l+p-1} \widetilde{\mathcal{A}}_{\mu-l-p}\left(k_{1}, \ldots, k_{r} ; 1\right) \lambda_{\mu+p+1} \frac{(i \pi)^{\mu}}{\mu !} .
\end{aligned}
$$

Let $p=0$ and $l \geq 2$. Since $\mathcal{S}_{r}^{0}\left(\pi ; k_{1}, \ldots, k_{r} ; l ; 1\right)=0$, we have

$$
\mathcal{F}^{0}\left(k_{1}, \ldots, k_{r} ; l\right)=i^{-1} \sum_{\nu=0}^{[(l-2) / 2]} \widetilde{\mathcal{A}}_{2 \nu+1-l}\left(k_{1}, \ldots, k_{r} ; 1\right) \frac{(i \pi)^{2 \nu+1}}{(2 \nu+1) !} .
$$

Now we recall the following lemma.

Lemma 4.1 ([9], Lemma 7). Suppose $\left\{P_{m}\right\}$ and $\left\{Q_{m}\right\}$ are sequences which satisfy the relation

$$
\sum_{j=0}^{[m / 2]} P_{m-2 j} \frac{(i \pi)^{2 j}}{(2 j+1) !}=Q_{m}
$$

for any $m \in \mathbb{N}_{0}$. Then the relation

$$
P_{m}=-2 \sum_{\nu=0}^{m} \phi(m-\nu) \lambda_{m-\nu} Q_{\nu}
$$

holds for any $m \in \mathbb{N}_{0}$.

Now we give the proof of Theorem 1.1. Applying Lemma 4.1 with

$$
\begin{aligned}
P_{m} & =\widetilde{\mathcal{A}}_{-m-1}\left(k_{1}, \ldots, k_{r} ; 1\right) \lambda_{\sum_{\eta=1}^{r} k_{\eta}+r+m}, \\
Q_{m} & =\frac{1}{\pi} \mathcal{F}^{0}\left(k_{1}, \ldots, k_{r} ; m+2\right) \lambda_{\sum_{\eta=1}^{r} k_{\eta}+r+m},
\end{aligned}
$$


it follows from (4.2) and (4.4) that

$$
\begin{aligned}
& \widetilde{\mathcal{A}}_{-m-1}\left(k_{1}, \ldots, k_{r} ; 1\right) \\
& =-2 \sum_{\nu=0}^{m} \phi(m-\nu) \lambda_{m+\nu} \frac{1}{\pi} \mathcal{F}^{0}\left(k_{1}, \ldots, k_{r} ; m-\nu+2\right) \in \mathcal{M} \mathcal{T}_{r-1}
\end{aligned}
$$

for any $m \in \mathbb{N}_{0}$ with $m \equiv \sum_{\eta=1}^{r} k_{\eta}+r(\bmod 2)$. Furthermore let $k \in \mathbb{N}$ with $k \geq 2$ and $k \not \equiv \sum_{\eta=1}^{r} k_{\eta}+r(\bmod 2)$. Namely $\sum_{\eta=1}^{r} k_{\eta}+k$ and $r$ are of different parity. Applying (4.3) with $l=k-1$ and $p=1$, we have

$$
\begin{aligned}
& \zeta_{M T, r}\left(k_{1}, \ldots, k_{r} ; k\right)+\mathcal{F}^{1}\left(k_{1}, \ldots, k_{r} ; k-1\right) \\
& =\sum_{\nu=0}^{[(k-2) / 2]} \widetilde{\mathcal{A}}_{2 \nu-k}\left(k_{1}, \ldots, k_{r} ; 1\right) \frac{(i \pi)^{2 \nu}}{(2 \nu) !}
\end{aligned}
$$

because $\mathcal{S}_{r}^{1}\left(\pi ; k_{1}, \ldots, k_{r} ; k ; 1\right)=\zeta_{M T, r}\left(k_{1}, \ldots, k_{r} ; k\right)$. By combining $(4.2),(4.5)$ and (4.6), we obtain the proof of Theorem 1.1.

\section{REFERENCES}

[1] M. E. Hoffman, Multiple harmonic series, Pacific J. Math. 152 (1992), 275-290. MR1141796 (92i:11089)

[2] J. G. Huard, K. S. Williams and Z. Nan-Yue, On Tornheim's double series, Acta Arith. 75 (1996), 105-117. MR1379394 (97f:11073)

[3] K. Ihara, M. Kaneko and D. Zagier, Derivation and double shuffle relations for multiple zeta values, Preprints of the Max-Planck-Institut für Mathematik, 2004-100 (2004).

[4] K. Matsumoto, On the analytic continuation of various multiple zeta-functions, in "Number Theory for the Millennium II, Proc. of the Millennial Conference on Number Theory" M. A. Bennett et al. (eds.), A K Peters, 2002. MR1956262 (2004a:11094)

[5] K. Matsumoto, On Mordell-Tornheim and other multiple zeta-functions, Proceedings of the Session in Analytic Number Theory and Diophantine Equations (Bonn, January-June 2002), D. R. Heath-Brown and B. Z. Moroz (eds.), Bonner Mathematische Schriften Nr. 360, Bonn 2003, No. 25, 17 pp. MF 2075634

[6] L. J. Mordell, On the evaluation of some multiple series, J. London Math. Soc. 33 (1958), 368-371. MF 0100181(20:6615)

[7] M. V. Subbarao and R. Sitaramachandrarao, On some infinite series of L. J. Mordell and their analogues, Pacific J. Math. 119 (1985), 245-255. MR0797027 (87c:11091)

[8] L. Tornheim, Harmonic double series, Amer. J. Math. 72 (1950), 303-314. MR0034860 $(11: 654 a)$

[9] H. Tsumura, Combinatorial relations for Euler-Zagier sums, Acta Arith. 111 (2004), 27-42. MR 2038060(2005a:11140)

[10] H. Tsumura, On a class of combinatorial relations for the Mordell-Tornheim triple series, preprint, submitted for publication.

Department of Management Informatics, Tokyo Metropolitan College, Akishima, TOKYO 196-8540, JAPAN

E-mail address: tsumura@tmca.ac.jp

Current address: Department of Mathematics, Tokyo Metropolitan University, 1-1, Minami-

Osawa, Hachioji, Tokyo 192-0397, Japan

E-mail address: tsumura@comp.metro-u.ac.jp 